\title{
PRIMER HALLAZGO DE SERPULIDOS (Rotularia clymenoides) EN EL EOCENO DE COSTA RICA
}

\author{
Teresita Aguilar \& Mario Arias \\ Escuela Centroamericana de Geología, Universidad de Costa Rica, \\ Apdo. 35 - 2060 U.C.R. \\ Correo electrónico: aaguilar@cariari.ucr.ac.cr
}

(Recibido 24/4/1998: Aceptado 10/9/1998)

\begin{abstract}
This is the first finding of serpulids "Rotularia clymenoides" in Costa Rica. Although, this group is very common in the Caribbean Eocene deposits. Serpulids includes species with high environmental tolerance, like those that have ocurred during the geological history of Costa Rica, specially in the evolution of the Istmus: like high volcanic input and inconsistent substrata composed by clastic material.as is documented in the sedimentary basins of Costa Rica.
\end{abstract}

RESUMEN: Se reporta el primer hallazgo en Costa Rica de serpúlidos: "Rotularia clymenoides”, en una secuencia sedimentaria que aflora a lo largo de la quebrada Concha. Este género es común en los depósitos eocénicos del Caribe. Fueron organismos con gran habilidad para colonizar ambientes de condiciones adversas, tales como: fuerte influencia volcánica, sustratos inestables con alto contenido de materiales clásticos, factores que imperaron durante la evolución y desarrollo del Istmo Centroamericano como se evidencia en el relleno de las cuencas sedimentarias de Costa Rica.

\section{INTRODUCCIÓN}

Durante un recorrido por la quebrada Concha, (coordenadas $187.55 \mathrm{~N}, 522.65 \mathrm{E}$ ), hoja topográfica Caraigres, escala 1:50.000 del Instituto geográfico Nacional; Fig. 1), se encontraron unas areniscas con restos de Serpúlidos de muy buena preservación. Con el fin de determinar su afinidad, se hicieron estudios de su superficie externa y de cortes y secciones pulidas. El material analizado se encuentra en la colección de fósiles de la Escuela Centroamericana de Geología, Universidad de Costa Rica, bajo los códigos: CF-5111 y CO-297.

Este es el primer reporte de Serpúlidos en Costa Rica, lo cual es de importancia debido a su valor en correlación estratigráfica (ya han sido usados en aplicaciones bioestratigráficas en la Antártica por Macellari, 1984). Además por ser organismos oportunistas, pueden encontrarse en rocas depositadas bajo condiciones ecológicas díficiles: volcanismo muy fuerte, 
sustratos inconsolidados e inestables, aguas someras y ambientes de energía moderadamente alta, similares a las condiciones que reinaron durante la mayor parte de la evolución y desarrollo del istmo centroamericano sur.

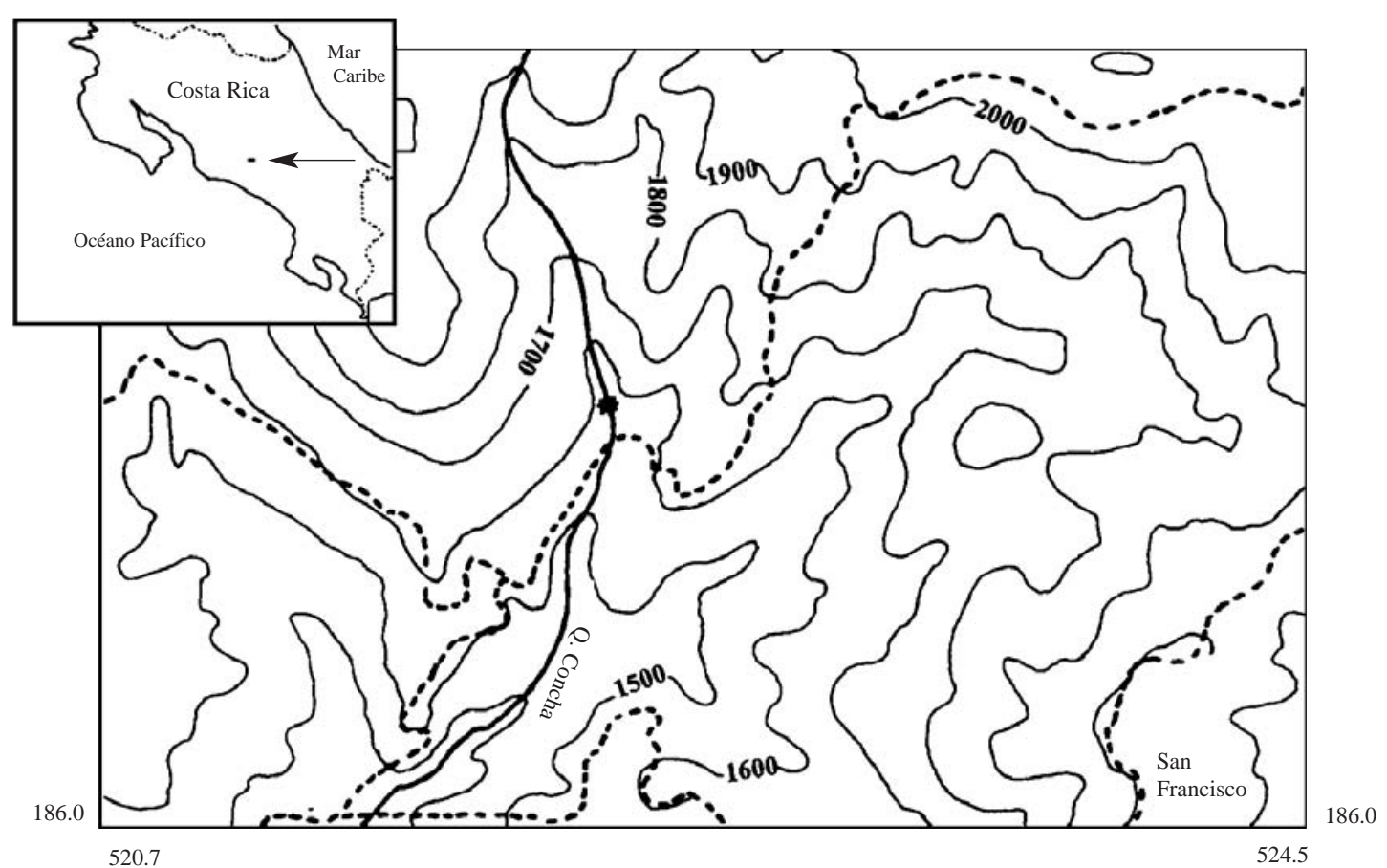

Fig. 1: Ubicación de la localidad en la quebrada Concha.

\section{MARCO GEOLÓGICO}

En las inmediaciones de la quebrada Concha se han realizado varios estudios geológicos regionales y locales, destacándose los siguientes:

Hayes, en 1899 (Hoffstetter et al., 1960), propone el nombre de Formación Brito para una secuencia de arenitas calcáreas, margas, areniscas con intercalaciones tobáceas, lutitas pardas con restos de plantas, brechas fosilíferas y calizas con macroforaminíferos, a las cuales les asigna una edad eocena. Posteriormente Dengo (1962) incluye esta secuencia en el Eoceno Medio y Superior. El estratotipo de la Formación Brito se definió en Nicaragua, y se correlacionó con La Unidad Cajón en Costa Rica, asignándole una edad de Eoceno Superior a partir de los rangos estratigráficos de macroforaminíferos determinados por Malavassi (1961).

Madrigal (1982), encuentra en zonas aledañas a la quebrada Concha una serie de lutitas, areniscas, vulcarenitas y calizas arrecifales y las correlaciona con la Formación Brito, destacando que las arenitas son dominantes, con un leve contenido de material calcáreo, su granulometría varía de fina a media, además reporta la presencia de macroforaminíferos.

Rivier \& Calvo (1988), definen la Unidad Parrita, precisamente en la quebrada Concha, como una sección carbonatada que presenta intercalación de facies detríticas, volcano-sedimentarias, con conglomerados y areniscas gruesas. En 
la base, las calizas descansan sobre una serie de lutitas negras, areniscas finas arcillosas y lutitas localmente compactadas y silicificadas. El contacto superior presenta una zona de transición de 2-3 metros de calizas arenosas, sobreyacidas por las areniscas volcánicas, calcáreas, con macroforaminíferos y areniscas tobaceas. Además, realizan un perfil esquemático en el que representan paquetes intercalados de areniscas masivas, color gris, con laminación gruesa y con horizontes conglomerádicos.

Denyer \& Arias (1991), elevan la Unidad Parrita al rango de formación, denominándola Formación Parritilla, la cual está compuesta por calizas bioclásticas grises (son destacables los macroforaminíferos) en estratos decimétricos y con intercalaciones métricas de areniscas y lutitas masivas de color gris.

Sprechmann, et al (1994), integra la Formación Parritilla en la Formación Fila de Cal, la cual incluye a todos los sistemas carbonatados, que tipifican plataformas y rampas carbonatadas con macroforaminíferos y algas calcáreas. Representa la época clímax de la sedimentación carbonatada, de la región centroamericana meridional, en la cual los mares neríticos fueron eminentemente carbonatados. Dentro de esta formación se incluyen los sedimentos del Eoceno Medio-Superior.

\section{Secuencia estratigráfica de la quebrada Concha}

Se realizó una columna estratigráfica en el margen derecho de la quebrada Concha, la cual se resume en la figura 2. Está compuesta por rocas basálticas del basamento local y regional, correlacionadas con el Complejo de Nicoya cuya edad en la zona, se puede considerar Cretácica, con base en dataciones de intercalaciones pelágicas inter-almohadillas (Alfaro, 1988; Denyer \& Arias, 1991).

Sobreyaciendo discordantemente se encuentra un paquete sedimentario que consiste de calizas con intercalaciones de estratos y lentes arenosos . Esta secuencia se correlaciona con la Formación Fila de Cal (Brito sensu Dengo,
1962; Parritilla sensu Denyer \& Arias, 1992), de edad Eoceno Medio - Superior (Sprechmann, et. al., 1993)

Las intercalaciones arenosas, tienen granulometría de fina a media, son de composición volcaniclástica con cemento carbonatado. El espesor varía entre 0,5 y $2 \mathrm{~m}$, en general el tamaño presenta una tendencia granocreciente o C. U. (coarsening upward).

Microscópicamente presentan porosidad interparticular e intraparticular, fábrica isotrópica, contacto por grano y en algunos casos por matríz, los granos son predominantemente muy angulares, es pobremente seleccionada e inmadura texturalmente.

Sus principales componentes son:

- Aloquímicos: 30\%, pellets, macroforaminíferos y algas?

- Ortoquímicos: $20 \%$, calcita y esparita.

- Terrígenos: $50 \%$, distribuidos de la siguiente manera:

Cuarzo: $3 \%$

Feldespatos: $25 \%$

Matriz: 20\%

Oxidos de hierro y clorita: $2 \%$

El nombre petrográfico asignado es Wacke Arcósica Bioclástica.

Precisamente en estas intercalaciones arenosas se hallaron los Serpúlidos (Rotularia clymenoides), junto con otros restos fósiles, entre ellos foraminíferos y fragmentos de moluscos.

La secuencia continúa con estratos de caliza, de color gris, con óxidos de hierro, buena compactación, estratificación métrica. Se determinaron algunos restos fósiles como: algas, oncoides, fragmentos de conchas y erizos.

El perfil levantado concluye con un paquete arenoso, con tendencia de tamaño de grano del tipo granodecreciente o F.U. (finning upward), con granulometrías desde gruesas hasta finas, las cuales se caracterizan por presentar buena selección y estructuras sedimentarias como laminación paralela e inclinada. En el techo de este paquete se encuentran intercalaciones de lentes centimétricos de conglomerados. 

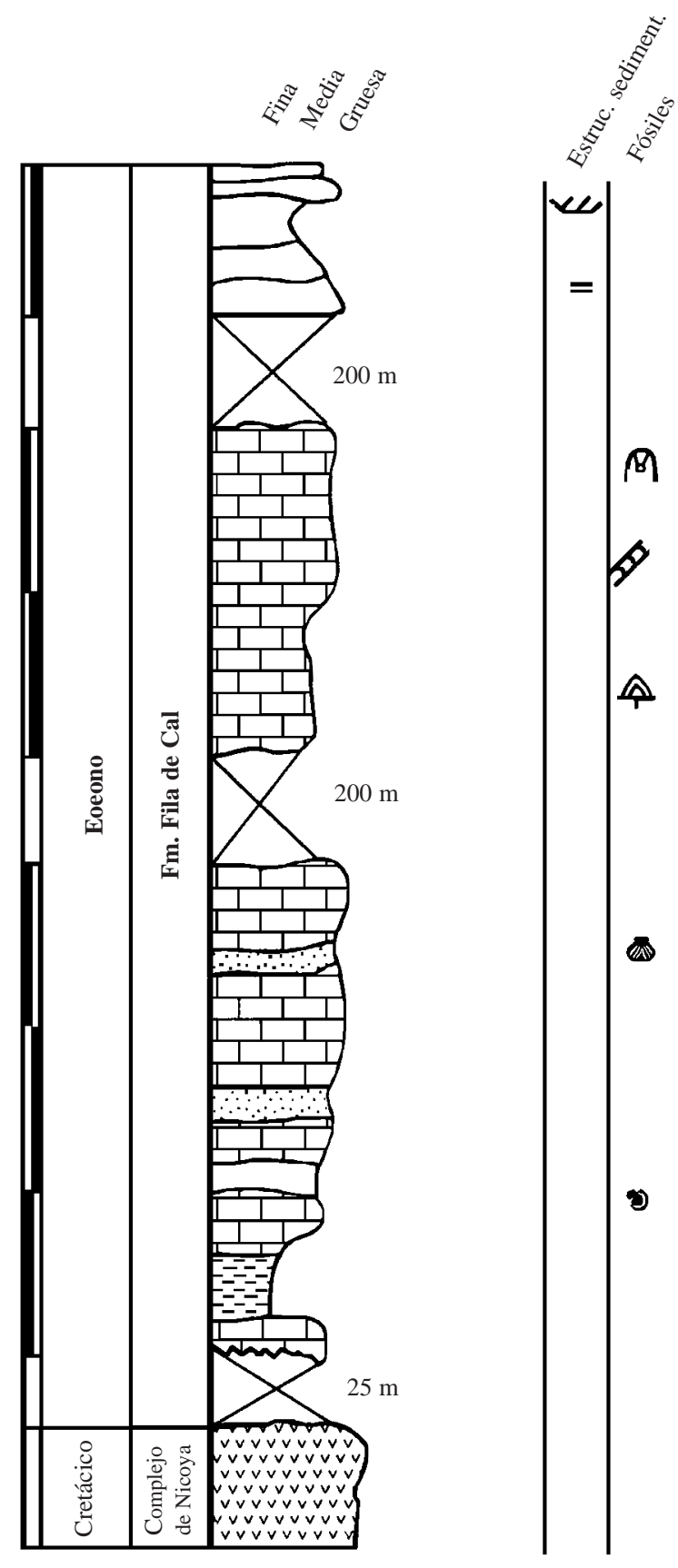

Intercalaciones de areniscas y conglomerados verdones

Paquetes de areniscas bien sorteados

\section{SIMBOLOGIA}

$=$ Laminación

- paralela

e Laminación

Caliza gris oscuro, muy

resistente; estratificación métrica
s. Algas
月 Oncoides
A Erizo
Moluscos
D Serpúlidos
No observado
E- Lutitas
E: Arenas
0 - Conglomerados
도 Caliza

Caliza gris con pátines de oxidos de hierro y algunos líticos

Caliza con paquetes arenosos intercalados, que presentan fuerte lixiviación de carbonatos

Nivel con serpúlidos

Intercalación de lutitas verdosas entre paquetes de caliza

Basaltos

Fig. 2: Columna estratigráfica de un sector de la quebrada Concha. 


\section{PALEOECOLOGÍA}

Los serpúlidos son anélidos de vida sedentaria, que habitan en el interior de un tubo segregado por su epidermis. El tubo puede ser de naturaleza orgánica, gelatinoso o coriáceo, otras veces pueden tener partículas minerales aglutinadas o puede ser calcáreo, lo cual aumenta considerablemente su potencial de fosilización (Meléndez, 1977).

La mayoría presentan un enrollamiento espiral, sin embargo, no existe una posición clara en cuanto a la dirección real de enrollamiento, aunque en la literatura se usa con más frecuencia (por analogía con los gastrópodos), orientar la porción cónica inicial de Rotularia con el ápice en la parte más superior (Savazzi, 1995). Los dos lados umbilicales se reconocen como: libre y cementado. Muchos especímenes adultos presentan además, un tubo apical, lo cual se interpreta como una evidencia de que vivieron enterrados bajo la superficie (Macellari, 1984).

Los ejemplares estudiados fueron encontrados en una arenisca calcáreea, muy inmadura, con feldespatos y cuarzos muy angulosos, lo cual indica un fuerte volcanismo en la zona, mientras ocurría la depositación. En este sustrato, son po$\cos$ los organismos que pueden subsistir, siendo $R$. clymenoides muy abundante, se deduce que la especie fue oportunista, capaz de colonizar un sustrato inhóspito.

\section{DESCRIPCIÓN SISTEMÁTICA}

\author{
Phylum Annelida Lamarck, 1809 \\ Clase Polichaeta Grübe, 1850 \\ Familia Serpulidae Burneister, 1837 \\ Subfamilia Spirorbinae Chamberlein, 1919 \\ Género Rotularia Defrance
}

Especie tipo: Rotularia spirulaea (Lamarck, 1818:366, designación posterior Wrigley, 1951:184)

Este género presenta una amplia variación morfológica (Ball, 1960).

\section{Rotularia (Rotularia) clymenoides GUPPY}

Los ejemplares estudiados tienen forma de tubo enrollado helicoidalmente, son discoidaleslenticulares y comprimidos (Fig. 3). Presentan de 3 a 4 vueltas aplastadas. Las primeras vueltas presentan un enrollamiento trocospiral bajo, las últimas están enrrolladas en el mismo plano (Fig. 4).

La superficie externa presenta líneas de crecimiento sinu-radiadas, con una carina (qui1la) periférica (Fig.3).

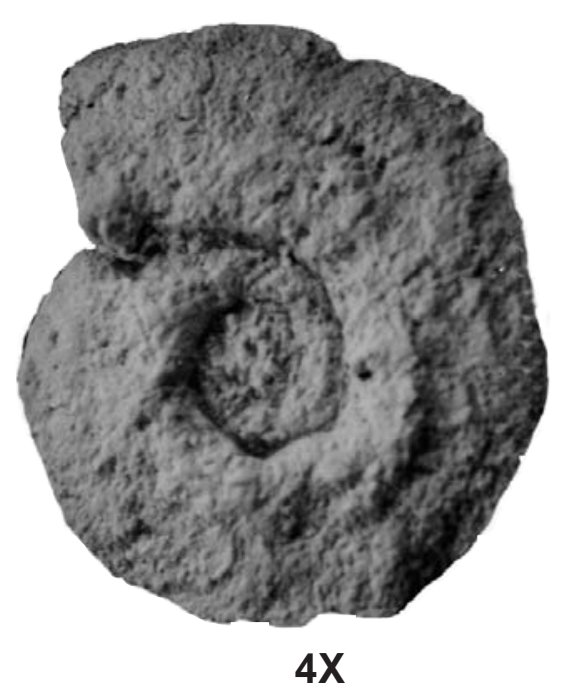

Fig. 3: R. clymenoides, mostrando la escultura y la quilla periférica.

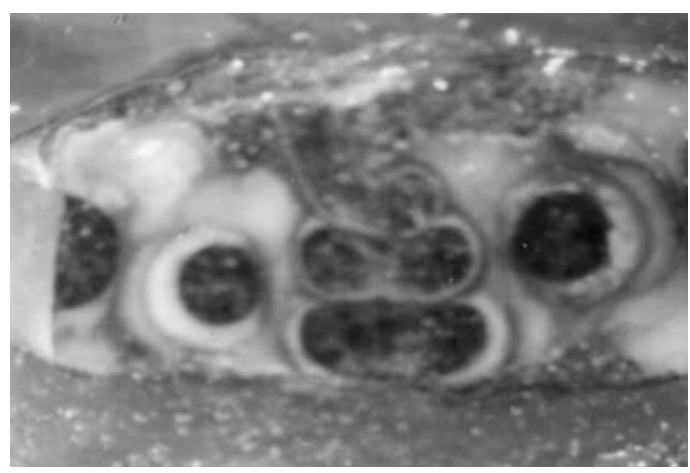

Fig. 4: Corte transversal que presenta el enrollamiento de las vueltas: las primeras trocoespirales y las últimas en un mismo plano. 
Los ejemplares encontrados no permiten observar bien la abertura, aunque parece que el diámetro disminuye con respecto al resto del tubo. El lumen es oblongo en sección transversal, principalmente cerca de la abertura. En el lado ventral presentan una leve concavidad.

\section{Observaciones:}

Nuestros ejemplares difieren de R. clymenoides (sensu Guppy, 1866) en el contorno más liso, mientras que el ejemplar figurado por Guppy, lámina XXVI, figura 10, presenta un contorno ondulado bien marcado. Esto podría deberse a que nuestros ejemplares sean juveniles (la mayoría son de tamaños muy pequeños, menores de $6 \mathrm{~mm}$, y los ejemplares más grandes, con un diámetro de $13 \mathrm{~mm}$, presentan una especie de ondulaciones en la parte más anterior). Otra posibilidad es que los ejemplares sean variantes ecológicas de $R$. clymenoides.

\section{Modo de vida:}

Los Rotularia no tienen parientes recientes con los que se puedan comparar, por eso se dificulta conocer sus requerimientos ambientales.

Generalmente se encuentran Rotularia en sedimentos bioturbados y son más abundantes entre más bioturbados sean los sedimentos. Esto indica su preferencia por un alto contenido de lodo en el sedimento, lo cual implica que fueron comedores de depósito o sedimentívoros. Para vivir en ambientes de alto contenido de lodo, poseían la quilla, la cual aumentaba la estabilidad de la concha y probablemente reducían el riesgo de hundirse en el sustrato. Otras formas utilizaron el tubo apical que se proyectaba sobre la superficie flocular del fondo lodoso. Contaban con un sistema de alimentación complicado (Macellari, 1984). Sin embargo, no hay datos suficientes para asegurar si Rotularia se alimentaba por suspención o eran comedores de detrito.

Según Macellari (1984), los Rotularia se comportan como un grupo oportunista, colonizando áreas ecologicamente díficiles. Savazzi (1995), indica que los Rotularia yacian sobre la superficie del sedimento con un lado expuesto y frecuentemente se daban vuelta, por lo cual presentaban un engrosamiento de la concha que las hacía más resistentes a la energía del medio y además reducía las posibilidades de ser depredados.

\section{Estructura de la concha:}

El tubo es calcítico. Presenta una capa interna delgada y sin estructura y una capa externa gruesa con estructura laminar.

\section{Distribución estratigráfica:}

El género Rotularia aparece en el Jurásico (Liásico Superior) y se extiende hasta el Eoceno (u ? Oligoceno Inferior) y tiene distribución mundial. La especie $R$. clymenoides, es típica del Eoceno del Caribe.

\section{Importancia del hallazgo:}

Es la primera vez que se reporta en América Central meridional el género Rotularia, el cual tiene amplia distribución a nivel mundial y ha sido reportado para rocas del Eoceno del Caribe. Este género tiene valor bioestratigráfico. Por lo que su presencia en Costa Rica es de gran importancia, debido a las condiciones geológicas que dieron origen a las rocas que conforman el Istmo Centroamericano Sur. Siendo ésta una zona de gran actividad tectónica y volcánica, la escasez de fósiles con valor bioestratigráfico, hace que especies como la que se ha descrito, amplien las posibilidades de correlación dentro del área de estudio y con la región centroamericana y Caribe.

\section{AGRADECIMIENTOS}

El hallazgo tuvo lugar durante la realización de una gira de Estratigrafía, en el I ciclo de 1994. El estudio se enmarca en el proyecto Análisis Geoestructural comparado de Costa Rica: 113-90-071. Deseamos agradecer la ayuda brindada por el Dr. Rudolf Fischer y el Dr. Manfred Jäger, en la identificación del material y el aporte de literatura adecuada para su estudio. Agradecemos además, la colaboración de los geólogos Percy Denyer y Sergio Hernández por la realización de fotografías y secciones delgadas y de Sergio Rojas por la elaboración de las figuras. Este trabajo es una contribución al Proyecto 301: Paleógeno de América del Sur. 


\section{REFERENCIAS}

ALFARO, A., 1988: Geología del área de Teruel de Acosta, comprendida entre la fila San Jerónimo y la fila Coyolar, cantón de Acosta, Costa Rica.- 36 págs; Escuela Centroamericana de Geología, Universidad de Costa Rica, Inf. Campaña Geológica.

DENGO, G, 1962: Estudio geológico de la región de Guanacaste, Costa Rica". - 122 págs; Instituto Geográfico de Costa Rica; San José, Costa Rica.

DENYER, P. \& ARIAS, O., 1991: Estratigrafía de la Región Central de Costa Rica.- Rev. Geol. América Central, 12: 1-59.

GARDNER, J., 1939: Notes on fossils from the Eocene of the Gulf Province. - U.S. Geol. Surv., Prof. Paper, 193-B: 17-37.

GUPPY, R. J. L, 1866: On the relations of the Tertiary formations of the West Indies. Q. J. Geol. Soc. London, 22: 570-590.

HASS, W. H., HÄNTZSCHEL, W., FISHER, D.W., HOWELL, B.F., RHODES, F.H.T., MÜLLER, K.J. \& MOORE, R.C., 1962: Systematic descriptions. En: MOORE, R.C. (ed.): Treatise on Invertebrate Paleontology. Part W, Miscellanea.- University of Kansas Press:W156-W161, Lawrence.

HOFFSTETTER, R., DENGO, G. DIXON, G., MEYER-ABICH, H., WEYL, R., WOODRING, W.P. \& ZOPPIS-BRACCI, L., 1960: Lexique Stratigraphique International, (5),America Latina, fasc.2a: Amerique Centrale, Paris:223-306.

JÄGER, M., 1993: Danian Serpulidae and Spirorbidae from NE Beogium and SE Netherlands: K/T Boundary Extinction, survival and origination patterns. - Contr. Tert. Quatern. Geol. 29(3-4): 73-137.

MACELLARI, C. E., 1984: Revision of serpulids of the genus Rotularia (Annelida) at
Seymour Island (Antarctic Peninsula) and their value in stratigraphy. - J. Paleont., 58(4): 1098-1116.

MADRIGAL, R. 1982: Informe Geológico Regional. - En: Informe Preliminar del Proyecto Hidroeléctrico Pirrís.- 38 págs Departamento de Programas de Generación, Oficina de Proyectos Hidroeléctricos, ICE, San José.

MALAVASSI., E., 1960: Algunas localidades de Costa Rica con foraminíferos grandes.- 5 págs; MEIC, Inf. Téc. Notas geol., 1(3), San José.

MELENDEZ, B., 1977: Paleontología. Tomo I. Parte general e invertebrados. - 715 págs.; Paraninfo, Madrid.

PIVETEAU, J., 1952: Traité de Paleontologie. Tome II: Problèmes d'adaptation et de Phylogénèse. - 790 págs.; Masson et Cie, Paris.

RIVIER, F. \& CALVO, C., 1988: Terciario del Sur del Valle Central: sección estratigráfica del Cerro Caraigres, Provincia de San José, Costa Rica. - Rev. Geol. América Central, 9: 61-74.

SPRECHMANN, P., (ed) 1984: Manual de Geología de Costa Rica, (1), Estratigrafía. 320 págs.; Editorial UCR, San José.

SPRECHMANN P., ASTORGA, A., FERNÁNDEZ, A. \& CALVO, C., 1993: Cuadro Sinóptico de Correlación Estratigráfica de las Cuencas Sedimentarias de Costa Rica. En: Memoria IV Congreso Geológico Nacional, Col. Geol. C.R. 7-16.

SPRECHMANN P., ASTORGA, A. CALVO, C. \& FERNÁNDEZ, A., 1994: Stratigraphic chart of the sedimentary basins of Costa Rica, Central América. - En: Seyfried, H. \& Hellmann, W. (eds.): Geology of and Evolving Island Arc. - Profil, 7: 427-433, Stuttgart, Germany. 